\title{
Diet induces hepatocyte protection in fatty liver disease via modulation of PTEN signaling (Review)
}

\author{
YUKA IKEDA, MUTSUMI MURAKAMI, YUKIE NAKAGAWA, \\ AI TSUJI, YASUKO KITAGISHI and SATORU MATSUDA \\ Department of Food Science and Nutrition, Nara Women's University, Nara 630-8506, Japan
}

Received April 10, 2019; Accepted July 11, 2019

DOI: $10.3892 /$ br.2020.1299

\begin{abstract}
Fatty liver disease (FLD) is characterized by accumulation of excess fat in the liver. The underlying molecular mechanism associated with the progression of the disease has been in elusive. Hepatocellular demise due to increased oxidative stress resulting in an inflammatory response may be a key feature in FLD. Recent advances in molecular biology have led to an improved understanding of the molecular pathogenesis, suggesting a critical association between the PI3K/AKT/PTEN signaling pathway and FLD. In particular, PTEN has been associated with regulating the pathogenesis of hepatocyte degeneration. Given the function of mitochondria in reactive oxygen species (ROS) generation and the initiation of oxidative stress, the mitochondrial antioxidant network is of interest. It is vital to balance the activity of intracellular key molecules to maintain a healthy liver. Consequently, onset of FLD may be delayed using dietary protective agents that alter PTEN signaling and reduce ROS levels. The advancement of research on dietary regulation with a focus on modulatory roles in ROS generation and PTEN associated signaling is summarized in the current study, supporting further preventive and therapeutic exploration.
\end{abstract}

Correspondence to: Professor Satoru Matsuda, Department of Food Science and Nutrition, Nara Women's University, Kita-Uoya Nishimachi, Nara 630-8506, Japan

E-mail: smatsuda@cc.nara-wu.ac.jp

Abbreviations: ALD, alcoholic liver disease; ASH, alcoholic steatohepatitis; DHA, docosahexaenoic acid; FLD, fatty liver disease; NAFLD, non-alcoholic fatty liver disease; NASH, non-alcoholic steatohepatitis; PIP3, phosphatidylinositol 3,4,5-triphosphate; PPAR, peroxisome proliferator-activated receptor; PUFA, polyunsaturated fatty acid; ROS, reactive oxygen species; SOD, superoxide dismutase

Key words: PTEN, reactive oxygen species, fatty liver, non-alcoholic fatty liver disease, non-alcoholic steatohepatitis, alcoholic liver disease, alcoholic steatohepatitis, cell signaling

\section{Contents}

1. Introduction

2. ROS involvement in the pathogenesis of fatty liver disease

3. PTEN in the anti-oxidative machinery

4. Diet and hepatocyte protection

5. Conclusions

\section{Introduction}

The liver is an organ performing vital functions (1). Hepatic disorders can affect the physiological and biochemical functions of the body. Fatty liver disease (FLD) is the prevalent form of chronic liver diseases that constitutes a range of disorders, starting with steatosis progressing to advanced stages, such as steatohepatitis, liver cirrhosis and hepatocellular carcinoma (HCC) (2). The origin of FLD involves nonalcoholic (NA) FLD and alcoholic liver disease (ALD (Fig. 1). In both cases, a complex process may cause the fatty liver in response to a variety of oxidative stress conditions (3). NAFLD is characterized by accumulation of excess fats in the liver of individuals unrelated to alcohol consumption; it is a progressive disease leading to irreversible liver injury (4). Non-alcoholic steatohepatitis (NASH) is an advanced stage of NAFLD, characterized by hepatic steatosis, ballooning injury and non-bacterial inflammation with or without fibrosis (5). The pathogenesis of the progression from NAFLD to NASH coincides with metabolic disorders that cause hepatosteatosis, and further progression to steatohepatitis is due to additional cellular processes, including mitochondrial injury, excess oxidative stress and inflammation (6). Genomic instability is one stage of hepatic carcinogenesis (7). Growing evidence suggests a key function of oxidative stress caused by the generation of reactive oxygen species (ROS) in the progression of FLD (Fig. 1) $(8,9)$. An additional influence in the progression from steatosis to steatohepatitis is the sensitization of hepatocytes to oxidative stress and cell apoptosis (10). As mitochondria serve a central role in the control of ROS generation and modulate the sensitivity to the cell apoptosis signaling pathway, mitochondrial function may be a key regulator in the development of steatohepatitis (11). Under normal conditions, living cells maintain a balance between ROS formation and quenching (12). 
An increasing incidence and prevalence worldwide mean that NAFLD has developed into a serious public health problem (13). As the underlying mechanism of FLD remains elusive and no effective therapeutic treatment has been established to date. It is suggested that excess uptake of free fatty acids from food leads to an early pathogenesis (14). Exercise is a simple therapeutic intervention; however, it was determined to be insufficient to relief symptoms (15). Type 2 diabetes, hypertriglyceridemia and obesity are frequently associated with NAFLD and NASH, while alcoholic steatohepatitis (ASH) is associated with alcohol abuse (16). ASH and NASH are intermediate stages of FLD, which can develop into cirrhosis or HCC. PTEN, a tumor suppressor, has been valuable in elucidating the pathways that regulate carcinogenesis in FLD (13). Progression of a fatty liver is accelerated by regulation of the liver PTEN signaling pathway (17). As with certain of other liver diseases, FLD increases the risk of liver cancer with poor outcomes and limited therapeutic options (13). Hence, a highly specific and effective drug treatment for FLD is required. The current review focuses on oxidative stress that contributes to the limited therapeutic effects in the treatment of FLD.

\section{ROS involvement in the pathogenesis of fatty liver disease}

Oxidative stress is considered as an imbalance between the generation of oxidants, such as ROS, and the activity of antioxidants, suggesting that the excess generation of free radicals and/or the modulation of antioxidant activity result in the accumulation of oxidative stress. Increased oxidative stress has harmful effects on cell functions that contribute to various diseases, including FLD (18). The imbalance in redox homeostasis is associated with the development of various tissue injuries, including brain, heart and bone (19). Cellular ROS increases via a certain pathways and mitochondrial damage. Mitochondria are the major intracellular site of oxygen consumption and are a source of ROS. Accordingly, alterations in mitochondrial function serve a significant role in the generation of ROS, which has been recognized to contribute to the development of ASH and NASH (11). Mitochondrial dysfunction is a contributor to ALD and this disease is linked to mitochondrial DNA fragmentation associated with active alcohol consumption (20). ROS are a group of oxygen-radical-containing molecules resulting from the metabolism of oxygen in the cells (21). Excessive concentrations of ROS result in macromolecular and genomic DNA damage, as well as cell death (22). In addition, elevated oxidative stress increases the risk of various cancer types (23). The formation of ROS during chronic inflammation is crucial to the progression of chronic liver diseases (24). ROS lead to a free radical chain-reaction in unsaturated fatty acids, a process called lipid peroxidation, generating toxic unsaturated aldehydes (25). The superoxide anion is the primary ROS generated in mitochondria and quenching of this compound is a critical step in preventing excessive oxidative stress. Additionally, ROS are physiologically important in signal transduction, cellular physiology, critical metabolic pathways and host defense $(26,27)$.

ROS are abundant free radicals in nature, and ROS production and genomic stability are affected by lifestyle factors (28). Certain environment-associated lifestyle factors, including tobacco and alcohol consumption, ionizing radiation, infection, inflammation and the aging process, cause oxidative stress (29). High blood glucose and excessive insulin further cause elevated ROS production (30). Hyperglycemia exacerbates FLD by elevating apoptosis via generation of excessive ROS (31). In addition, obese patients have shown significantly higher serum levels oxidative stress compared with non-obese controls (32). Intensive aerobic and anaerobic exercise increases oxidative damage (33). Strenuous exercise disturbs the antioxidant equilibrium by increasing ROS levels. However, regular exercise upregulates endogenous antioxidant levels and reduces oxidative damage (34). As continued exposure to uncontrolled oxidative stress is an initiator of various chronic diseases and cancer, cells have developed a range of antioxidant strategies, including enzymatic and non-enzymatic antioxidants (35). Maintaining healthy ROS level is indispensable for the conservation of healthy cells and particularly in mitochondria a balance of antioxidants is necessary to avoid oxidative stress. Superoxide dismutases (SODs) have a strong antioxidant role characterized by scavenging ROS, through the reaction of superoxide to hydrogen peroxide (36). SODs are the primary defense against cellular damage by oxidative stress and the breakdown of mitochondrial superoxide is performed by manganese SOD (SOD2) (37). In addition to antioxidants, cells use distinct oxidative damage-repair mechanisms to eliminate DNA damage (38).

\section{PTEN in the anti-oxidative machinery}

ROS have been shown to modulate various physiological processes, including the regulation of growth factor signaling. One mechanism by which ROS exert cellular effects is through the regulation of target molecules, including PI3K/AKT/PTEN (39). The PI3K/AKT/PTEN signaling pathway protects against damaging effects originating from high levels of insulin (40). In addition, the PI3K/AKT/PTEN signaling pathway regulates factors involved in cell survival and proliferation (Fig. 2) (40). PTEN is a dual specificity phosphatase, processing lipids and proteins, and it is a member of the protein tyrosine phosphatase family of phosphatases $(41,42)$. PTEN downregulates AKT activity through the conversion of phosphatidylinositol 3,4,5-triphosphate (PIP3) to phosphatidylinositol 4,5-bisphosphate (43). Cells lacking PTEN have higher levels of PIP3, a critical second messenger mediating signals from various growth factors, including insulin (44). PTEN is a tumor suppressor and is involved in oxidative stress and genomic damage induction (45). Inactivation of PTEN leads to prolonged AKT activation, increased mitochondrial respiration and increased ROS in mouse models (40). Downregulation, inhibition or deactivation of PTEN results in an increase of mitochondrial ATP production (46). Furthermore, PTEN is associated with the activation of the proteolytic cell apoptosis cascade through decreased activity of PI3K/AKT signaling (47).

Deficiencies of PTEN have been shown to promote NASH development (48). A hepatocyte-specific deletion of the PTEN gene exhibited an age-dependent development of liver steatosis and HCC $(49,50)$. In addition, animal models of PTEN haploinsufficiency exhibit hepatomegaly, increased liver lipogenic gene expression, including of peroxisome 


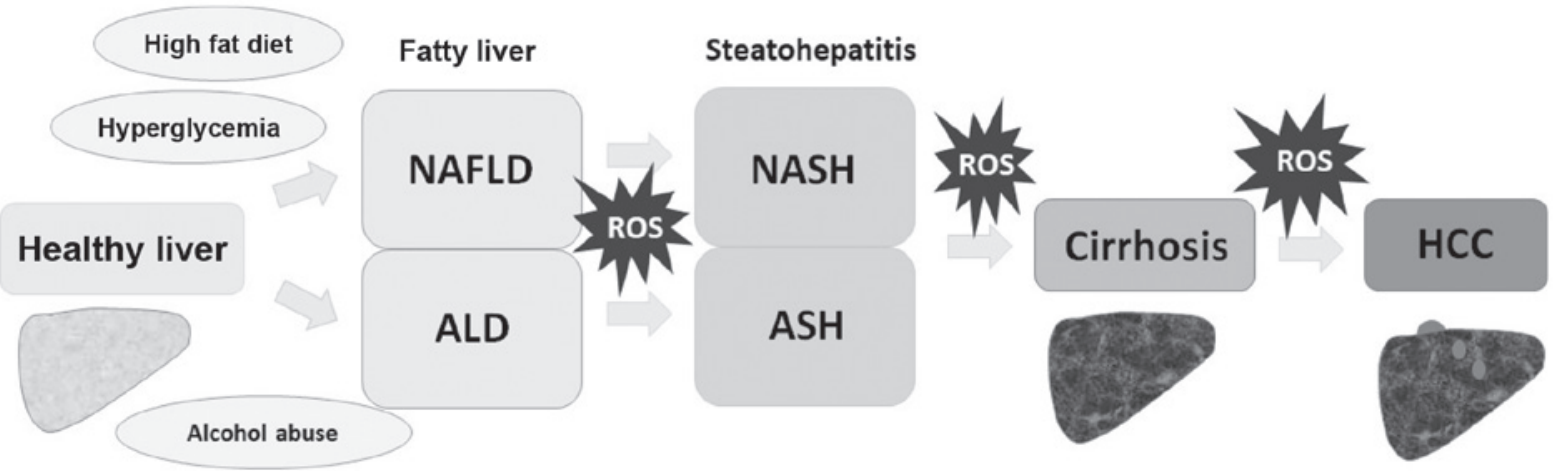

Figure 1. ROS affects the development in fatty liver diseases. Various roles of ROS in the development of steatohepatitis, including NASH and ASH, liver cirrhosis and HCC. Certain factors and stages were omitted for clarity. ROS, reactive oxygen species; HCC, hepatocellular carcinoma; ALD, alcoholic liver disease; ASH, alcoholic steatohepatitis; NAFLD, nonalcoholic fatty liver disease; NASH, nonalcoholic steatohepatitis.

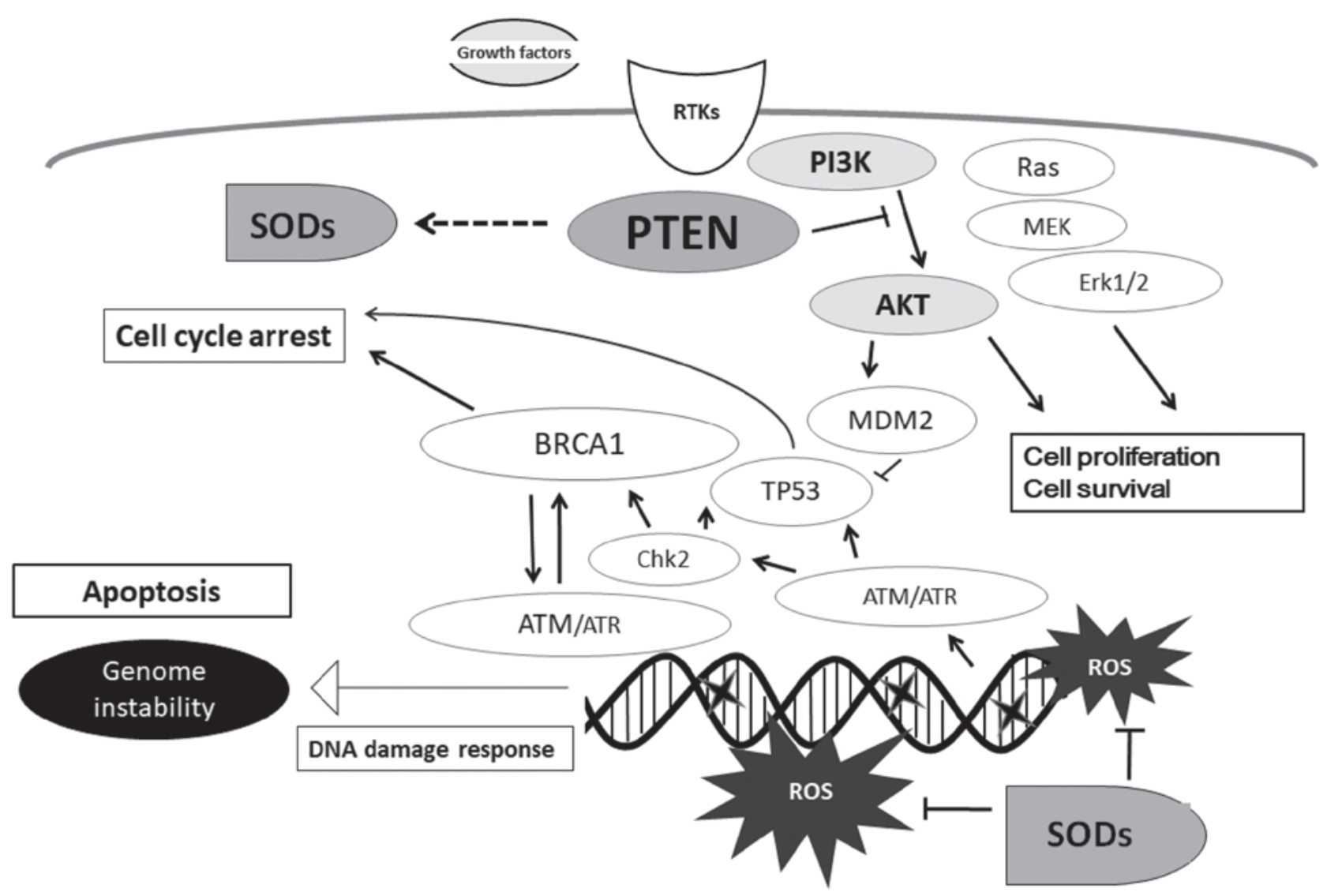

Figure 2. PTEN and oxidative DNA stress signaling pathways. Schematic representation of the regulatory pathways with exemplary molecules included; certain molecules were omitted for clarity. Solid arrows represent direct interaction; dashed arrows represent indirect interaction and the white head arrow refers to weak interaction. ROS, reactive oxygen species; SOD, superoxide dismutase; RTK, receptor tyrosine kinase.

proliferator-activated receptor (PPAR) $\gamma$ and hepatic lesions, symptoms that are analogous to NAFLD (51). PTEN deficiency in hepatocytes induces upregulation of PI3K/AKT signaling, following increased lipogenesis and decreased lipolysis (52). Consistently, PTEN knockout mice exhibit insulin hypersensitivity, constitutive lipogenesis and hepatomegaly (53). In humans, PTEN mutations have been described in association with insulin hypersensitivity and obesity (54). In patients with FLD, reduced expression of PTEN and upregulation of AKT have been observed in liver biopsies (55). In addition, patients with NASH have shown decreased expression of PTEN compared with healthy patients (56). A marked increase of PTEN in hepatocellular steatosis progresses the disease to steatohepatitis and fibrosis, and in certain cases HCC (57). A previous study described PTEN and obesity-associated disorders as risk factors for HCC (13).

\section{Diet and hepatocyte protection}

Various disease-protective factors have been suggested in epidemiological studies $(58,59)$. Dietary choices have been indicated to serve a role in liver protection (Fig. 3). Particularly, 


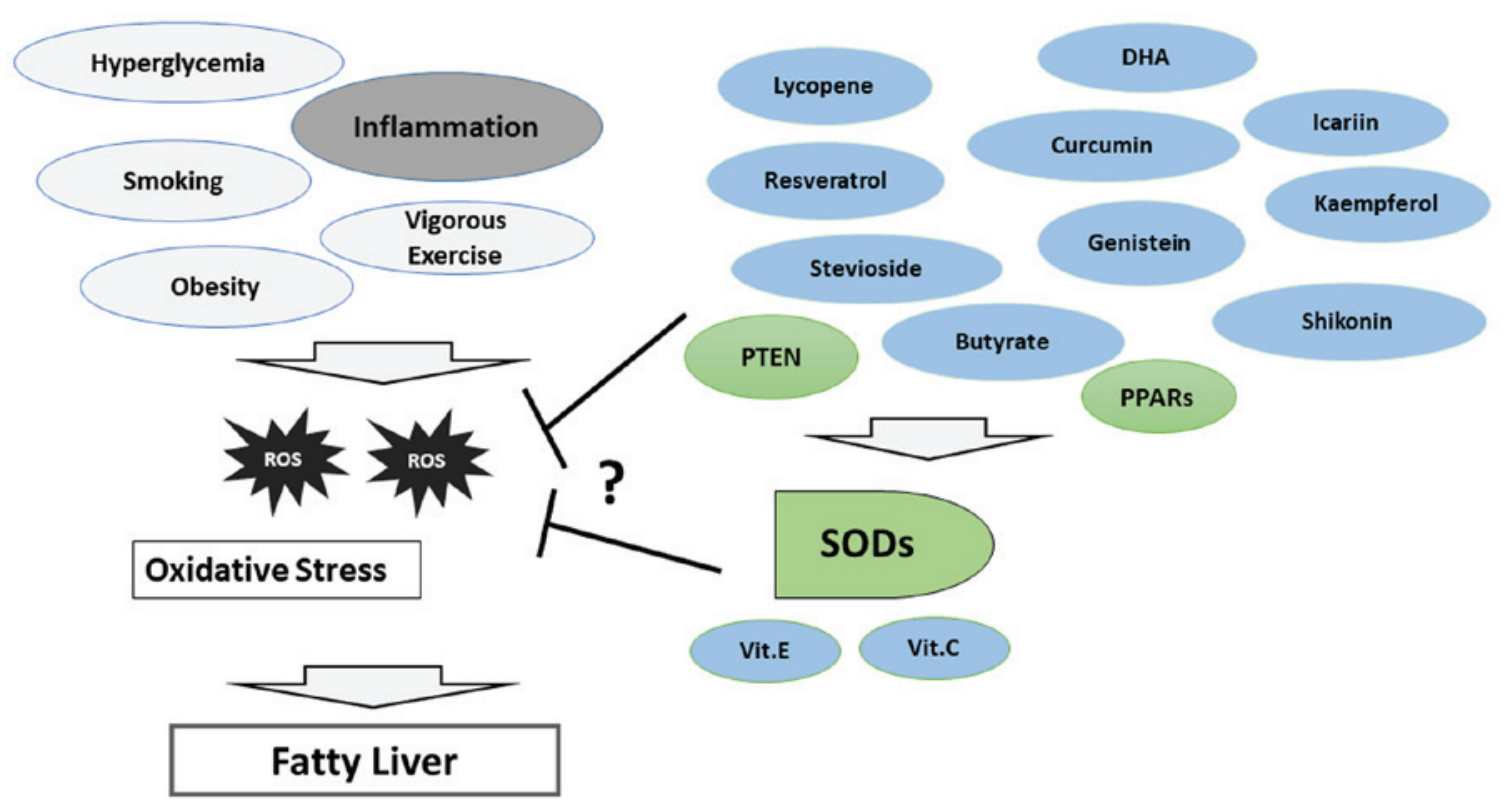

\section{Hepatocyte Apoptosis and/or Carcinogenesis}

Figure 3. Lifestyle and diet affect oxidative stress levels. Certain food ingredients and dietary components contribute to the prevention of fatty liver diseases via the modulation of PTEN and SOD activities and lifestyle choices can impact liver health. PPAR, peroxisome proliferator-activated receptor; SOD, superoxide dismutase; ROS, reactive oxygen species; DHA, docosahexaenoic acid; Vit, vitamin.

dietary choices that modify the PI3K/AKT/PTEN signaling pathway may prevent FLD or decrease the rate of disease progression (60). Certain plants and fruits are promising targets (61-67).

Curcumin, an active ingredient derived from the root of Curcuma longa used as culinary turmeric, exhibits therapeutic potential for the treatment of diabetes and various types of cancer (62). Curcumin has strong antioxidant and anti-inflammatory effects and exhibits liver protective properties in an animal model (61). This protection is mediated by the PI3K/AKT/PTEN signaling pathway (63). Shikonin is a natural compound extracted from the roots of Lithospermum erythrorhizon (64) that was shown to prevent hepatotoxicity by upregulation of the PI3K/AKT/PTEN signaling pathway in an animal model (65). Kaempferol is a flavonol present in several plants, including grapefruits and edible berries, which was shown to inhibit hepatocyte apoptosis and prevent acute liver failure in an animal study (66). Icariin is a prenylated flavonol glycoside from Epimedium koreanum, which downregulates PTEN expression following AKT overexpression in an animal model (67). An antihepatotoxic activity of Icariin has been demonstrated in carbon tetrachloride-induced hepatocytes (68). In addition, in an animal model, rosemary (Rosmarinus officinalis L.) was shown to have liver protection activity at various stages of liver damage (69). Certain to-date unknown components of rosemary inhibit PTEN expression in K562 myeloid cells (70) and rosemary promotes liver regeneration in an experimental injury model (69). In contrast, levels of PTEN are increased when treated with Ginsenoside, a class of natural steroid glycosides, which exhibit hepatoprotective effects against acute hepatotoxicity in mice (71). Furthermore, certain types of diet rich in fat or carbohydrates contribute to hepatocyte protection (72). Further exploration is required to establish whether the protective characteristics are associated with the PI3K/AKT/PTEN signaling pathway.

Antrodia camphorata is a common mushroom found and used in Asia that protects against liver injury through reducing mitochondrial ROS (73). The active ingredient Genistein inhibits ROS production by enhancing SOD activities in an animal model (74). Genistein protects hepatocytes against toxicity due to creating a resistance to oxidative stress (75). Lycopene, an antioxidant found in red fruits such as tomatoes, inhibits hepatocyte apoptosis by reducing ROS levels and inhibiting mitochondrial dysfunction, and may have the ability to prevent FLD (76). It has been suggested that dietary intake of copper chloride and/or copper sulphate stabilizes SOD activity in an animal model, indicating a potential therapeutic benefit for FLD (77). Expression of SOD is associated with PPAR activity (78). The grape antioxidant resveratrol and its analogs increase SOD mRNA and protein expression levels in vitro (79). Furthermore, increased expression of SOD2 has been detected after administration of grape juice to an animal model (80). Commercially available grape juice reduces oxidative damage in the liver of experimental rats (81). Stevioside from Stevia leaves, a natural sweetener, increases the expression of various SODs, including SOD2, in mice experiments (82). The antioxidant potential of stevia extracts from Stevia leaves has been reported in an experimental liver damage model (83). Butyrate, a short-chain fatty acid which can be prepared from various vegetables, increases the expression of SODs (84), protecting mice from an early development of NAFLD (85). Blueberry juice protects liver function by reducing mitochondrial oxidative stress through elevating SOD and suppressing ROS activity in an animal model (86). Supplementation of antioxidants may protect 
hepatocytes from oxidative damage. Antioxidant vitamins, including $\mathrm{C}$ and $\mathrm{E}$, have also presented hepatocyte protective effects in an animal toxicity model (87). Enhanced protection of liver membranes has been described for animals fed with a coenzyme Q10 (88). Consumption of vitamin E has been linked to regeneration of SODs in an animal model (89). In addition, a long-term diet rich in polyunsaturated fatty acid (PUFA) and/or docosahexaenoic acid (DHA) leads to lower oxidative damage to proteins. DHA supplementation has been suggested as a preventive approach for patients with ALD based on findings of an animal model (90). Perilla frutescens is a source of PUFA, with high levels present compared with other edible plants, and PUFA can be converted to DHA in the animal liver (91).

It is suggested that SODs, as well as dietary antioxidants, may offer hepatocyte protection against the progression of FLD (Fig. 3). However, the association between nutrient consumption and hepatocyte protection is complex and requires further investigation. In addition, the complexity of the human diet makes it challenging to examine distinctive effects. As presented, certain food or dietary components provide hepatocyte protection through signaling pathway modifications via modulation of specific activities. Furthermore, the microbiome has been described as a hallmark of various liver diseases (92). It has been reported that probiotics, including Lactobacillus, restores gut microbiota and alleviates liver injury in animal models (93). The proportion of Bacteroides has been described as markedly higher in patients with liver fibrosis compared with healthy controls, which influences NAFLD progression (94). Furthermore, probiotic administration has been associated with improved levels of liver markers of hepatic inflammation in patients with NAFLD (95). Accordingly, modulation of the gut microbiome presents a new therapeutic target in NAFLD treatment due to the distinct changes in the composition of gut microbiota. In the future, dietary approaches restoring gut microbiota may emerge as a therapy fields for FLD.

\section{Conclusions}

The development FLD and co-morbidities has severe effects on the liver and associated functions. There are no approved therapies for the treatment of FLDs. Therapeutic progress is limited by the poor understanding of the initiating steps of fat accumulation in the liver. Accordingly, potential endogenous modulators of the pathogenesis may provide tools for therapeutic intervention. Any conceivable therapeutic strategy should build on the observation that there are changes in key processes required for the cellular function. Therefore, properties of food ingredients may have certain hepatocyte protective potentials, which are facilitated through reduction of ROS production. To maintain normal cellular function, cells are required to escape excessive oxidative stress. This represents a rational basis for the development of dietary treatments for FLD. However, despite the various experimental observations using food ingredients, the precise mechanisms remain elusive and are therefore not suitable for clinical use. Additional mechanistic studies are required to understand detailed molecular mechanisms and to clarify if certain dietary intake is associated with improved hepatocyte survival.
Liver steatosis is associated with mitochondrial dysfunction and excessive mitochondrial ROS production. Although mitochondria are key in the maintenance of cell functions, they are also the main source of ROS. The dual function of ROS, as apoptosis triggers and performers in cell survival signaling, may determine the role of mitochondrial function in disease progression. An instrumental role of PTEN in hepatic carcinogenesis has been suggested for obese patients highlighting potential antioxidant involvement. It is imperative to exploit benefits from treatment in combination with chemical and medical modulators associated with the function of ROS and PTEN. Long-term clinical studies are further required to clarify distinct effects in the management of FLD to address therapeutic potential.

In conclusion, ROS and PTEN are involved in a pathogenesis of FLD and certain diets associated with PTEN signaling may contribute to disease prevention or progression through hepatocyte protection from apoptosis induced by oxidative stress.

\section{Acknowledgements}

Not applicable.

\section{Funding}

This work was supported by JSPS KAKENHI (grant no. JP18K17964) and the Nara Women's University in Japan.

\section{Availability of data and materials}

Not applicable.

\section{Authors' contributions}

YI and SM have contributed to the conception and design of the study. YI, MM, YN, AT, YK and SM participated in drafting and revising the article. All authors approved the final manuscript.

\section{Ethics approval and consent to participate}

Not applicable.

\section{Patient consent for publication}

Not applicable.

\section{Competing interests}

The authors declare that they have no competing interests.

\section{References}

1. Protzer U, Maini MK and Knolle PA: Living in the liver: Hepatic infections. Nat Rev Immunol 12: 201-213, 2012.

2. Liu A, Galoosian A, Kaswala D, Li AA, Gadiparthi C, Cholankeril G, Kim D and Ahmed A: Nonalcoholic fatty liver disease: Epidemiology, liver transplantation trends and outcomes, and risk of recurrent disease in the graft. J Clin Transl Hepatol 6: 420-424, 2018.

3. Sundaram V and Morgan TR: Will studies in nonalcoholic steatohepatitis help manage alcoholic steatohepatitis? Clin Liver Dis 23: $157-165,2019$. 
4. Eslam $\mathrm{M}$ and George $\mathrm{J}$ : Genetic insights for drug development in NAFLD. Trends Pharmacol Sci 40: 506-516, 2019.

5. Chalasani N, Younossi Z, Lavine JE, Diehl AM, Brunt EM, Cusi K, Charlton M and Sanyal AJ: The diagnosis and management of non-alcoholic fatty liver disease: Practice Guideline by the American Association for the Study of Liver Diseases, American College of Gastroenterology, and the American Gastroenterological Association. Hepatology 55: 2005-2023, 2012

6. Anstee QM, Reeves HL, Kotsiliti E, Govaere O and Heikenwalder M: From NASH to HCC: Current concepts and future challenges. Nat Rev Gastroenterol Hepatol 16: 411-428, 2019.

7. Karaman H, Karaman A, Donmez-Altuntas H, Bitgen N, Hamurcu Z, Oguz A and Karakukcu C: Investigation of genome instability in patients with non-alcoholic steatohepatitis. World J Gastroenterol 19: 5295-5301, 2013

8. Ma Z, Zhang Y, Li Q, Xu M, Bai J and Wu S: Resveratrol improves alcoholic fatty liver disease by downregulating HIF-10 expression and mitochondrial ROS production. PLoS One 12 e0183426, 2017.

9. Simões ICM, Fontes A, Pinton P,Zischka $\mathrm{H}$ and Wieckowski MR: Mitochondria in non-alcoholic fatty liver disease. Int J Biochem Cell Biol 95: 93-99, 2018.

10. Schattenberg JM and Schuppan D: Nonalcoholic steatohepatitis: The therapeutic challenge of a global epidemic. Curr Opin Lipidol 22: 479-488, 2011.

11. García-Ruiz C and Fernández-Checa JC: Mitochondrial oxidative stress and antioxidants balance in fatty liver disease. Hepatol Commun 2: 1425-1439, 2018

12. Poljsak B: Strategies for reducing or preventing the generation of oxidative stress. Oxid Med Cell Longev 2011: 194586, 2011.

13. Michelotti GA, Machado MV and Diehl AM: NAFLD, NASH and liver cancer. Nat Rev Gastroenterol Hepatol 10: 656-665, 2013.

14. Jacome-Sosa MM and Parks EJ: Fatty acid sources and their fluxes as they contribute to plasma triglyceride concentrations and fatty liver in humans. Curr Opin Lipidol 25: 213-220, 2014.

15. Keating SE, Hackett DA, George J and Johnson NA: Exercise and non-alcoholic fatty liver disease: A systematic review and meta-analysis. J Hepatol 57: 157-166, 2012.

16. Gruszewska E, Gudowska M, Wojtowicz E, Cylwik B, Szmitkowski M and Chrostek L: The higher prevalence of non-alcoholic versus alcoholic steatohepatitis in alcoholics. Clin Lab 61: 1769-1774, 2015.

17. Wang Z, Li N, Wang B and Lin J: Nonalcoholic fatty liver disease progression in rats is accelerated by splenic regulation of liver PTEN/AKT. Saudi J Gastroenterol 21: 232-238, 2015.

18. Flisiak-Jackiewicz M and Lebensztejn DM: Update on pathogenesis, diagnostics and therapy of nonalcoholic fatty liver disease in children. Clin Exp Hepatol 5: 11-21, 2019.

19. Knapik DM, Perera P, Nam J, Blazek AD, Rath B, Leblebicioglu B, Das H, Wu LC, Hewett TE, Agarwal SK Jr, et al: Mechanosignaling in bone health, trauma and inflammation. Antioxid Redox Signal 20: 970-995, 2014.

20. Smiles WJ, Parr EB, Coffey VG, Lacham-Kaplan O, Hawley JA and Camera DM: Protein coingestion with alcohol following strenuous exercise attenuates alcohol-induced intramyocellular apoptosis and inhibition of autophagy. Am J Physiol Endocrinol Metab 311: E836-E849, 2016

21. Uzelac L, Škalamera Đ, Mlinarić-Majerski K, Basarić N and Kralj M: Selective photocytotoxicity of anthrols on cancer stem-like cells: The effect of quinone methides or reactive oxygen species. Eur J Med Chem 137: 558-574, 2017.

22. Caputo F, Vegliante R and Ghibelli L: Redox modulation of the DNA damage response. Biochem Pharmacol 84: 1292-1306, 2012.

23. Barnes JL, Zubair M, John K, Poirier MC and Martin FL: Carcinogens and DNA damage. Biochem Soc Trans 46: 1213-1224, 2018

24. Mansouri A, Gattolliat $\mathrm{CH}$ and Asselah T: Mitochondrial dysfunction and signaling in chronic liver diseases. Gastroenterology 155: 629-647, 2018.

25. Medina-Navarro R, Duran-Reyes G, Diaz-Flores M, Hicks JJ and Kumate J: Glucose-stimulated acrolein production from unsaturated fatty acids. Hum Exp Toxicol 23: 101-105, 2004.

26. Futosi K, Fodor S and Mócsai A: Neutrophil cell surface receptors and their intracellular signal transduction pathways. Int Immunopharmacol 17: 638-650, 2013.

27. Banoth B and Cassel SL: Mitochondria in innate immune signaling. Transl Res 202: 52-68, 2018
28. Mena S, Ortega A and Estrela JM: Oxidative stress in environmental-induced carcinogenesis. Mutat Res 674: 36-44, 2009.

29. Venza I, Visalli M, Oteri R, Teti D and Venza M: Combined effects of cigarette smoking and alcohol consumption on antioxidant/oxidant balance in age-related macular degeneration. Aging Clin Exp Res 24: 530-536, 2012

30. Rains JL and Jain SK: Oxidative stress, insulin signaling, and diabetes. Free Radic Biol Med 50: 567-575, 2011.

31. Zhu W, Jia Q, Wang Y, Zhang Y and Xia M: The anthocyanin cyanidin-3-O- $\beta$-glucoside, a flavonoid, increases hepatic glutathione synthesis and protects hepatocytes against reactive oxygen species during hyperglycemia: Involvement of a cAMP-PKA-dependent signaling pathway. Free Radic Biol Med 52: 314-327, 2012.

32. Roh HT, Cho SY and So WY: Obesity promotes oxidative stress and exacerbates blood-brain barrier disruption after high-intensity exercise. J Sport Health Sci 6: 225-230, 2017.

33. He F, Li J, Liu Z, Chuang CC, Yang W and Zuo L: Redox mechanism of reactive oxygen species in exercise. Front Physiol 7: 486, 2016.

34. Smith Sonneborn J: Alternative strategy for Alzheimer's disease: Stress response triggers. Int J Alzheimers Dis 2012: 684283, 2012.

35. Farombi EO, Shyntum YY and Emerole GO: Influence of chloroquine treatment and Plasmodium falciparum malaria infection on some enzymatic and non-enzymatic antioxidant defense indices in humans. Drug Chem Toxicol 26: 59-71, 2003.

36. Johnson F and Giulivi C: Superoxide dismutases and their impact upon human health. Mol Aspects Med 26: 340-352, 2005.

37. Smith MR, Fernandes J, Go YM and Jones DP: Redox dynamics of manganese as a mitochondrial life-death switch. Biochem Biophys Res Commun 482: 388-398, 2017.

38. Turgeon MO, Perry NJS and Poulogiannis G: DNA damage, repair, and cancer metabolism. Front Oncol 8: 15, 2018.

39. Nakanishi A, Wada Y, Kitagishi Y and Matsuda S: Link between PI3K/AKT/PTEN pathway and NOX proteinin diseases. Aging Dis 5: 203-211, 2014.

40. Bankoglu EE, Tschopp O, Schmitt J, Burkard P, Jahn D, Geier A and Stopper H: Role of PTEN in oxidative stress and DNA damage in the liver of Whole-Body Pten Haplodeficient Mice. PLoS One 11: e0166956, 2016.

41. Lyu J, Yu X, He L, Cheng T, Zhou J, Cheng C, Chen Z, Cheng G, Qiu Z and Zhou W: The protein phosphatase activity of PTEN is essential for regulating neural stem cell differentiation. Mol Brain 8: 26, 2015.

42. Oganesian A, Poot M, Daum G, Coats SA, Wright MB, Seifert RA and Bowen-Pope DF: Protein tyrosine phosphatase RQ is a phosphatidylinositol phosphatase that can regulate cell survival and proliferation. Proc Natl Acad Sci USA 100: 7563-7568, 2003.

43. Carnero A and Paramio JM: The PTEN/PI3K/AKT pathway in vivo, cancer mouse models. Front Oncol 4: 252, 2014.

44. Chen CY, Chen J, He L and Stiles BL: PTEN: Tumor suppressor and metabolic regulator. Front Endocrinol (Lausanne) 9: 338, 2018.

45. Kitagishi $Y$ and Matsuda S: Redox regulation of tumor suppressor PTEN in cancer and aging (Review). Int J Mol Med 31: 511-515, 2013.

46. Liu Y, Yan J, Sun C, Li G, Li S, Zhang L, Di C, Gan L, Wang Y, Zhou R, et al: Ameliorating mitochondrial dysfunction restores carbon ion-induced cognitive deficits via co-activation of NRF2 and PINK1 signaling pathway. Redox Biol 17: 143-157, 2018.

47. Liu Y, Cao Y, Sun S, Zhu J, Gao S, Pang J, Zhu D and Sun Z: Transforming growth factor-beta1 upregulation triggers pulmonary artery smooth muscle cell proliferation and apoptosis imbalance in rats with hypoxic pulmonary hypertension via the PTEN/AKT pathways. Int J Biochem Cell Biol 77: 141-154, 2016.

48. Luo X, Liao R, Hanley KL, Zhu HH, Malo KN, Hernandez C, Wei X, Varki NM, Alderson N, Chu C, et al: Dual Shp2 and Pten deficiencies promote Non-alcoholic Steatohepatitis and genesis of liver tumor-initiating cells. Cell Rep 17: 2979-2993, 2016.

49. Piguet AC, Saran U, Simillion C, Keller I, Terracciano L, Reeves HL and Dufour JF: Regular exercise decreases liver tumors development in hepatocyte-specific PTEN-deficient mice independently of steatosis. J Hepatol 62: 1296-1303, 2015.

50. Gao M and Liu D: CRISPR/Cas9-based Pten knock-out and Sleeping Beauty Transposon-mediated Nras knock-in induces hepatocellular carcinoma and hepatic lipid accumulation in mice. Cancer Biol Ther 18: 505-512, 2017.

51. Eritja N, Arjó G, Santacana M, Gatius S, Ramírez-Núñez O, Arcal L, Serrano JCE, Pamplona R, Dolcet X, Piñol C, et al: Oral intake of genetically engineered high-carotenoid corn ameliorates hepatomegaly and hepatic steatosis in PTEN haploinsufficient mice. Biochim Biophys Acta 1862: 526-535, 2016. 
52. Trohatou O, Zagoura D, Orfanos NK, Pappa KI, Marinos E, Anagnou NP and Roubelakis MG: miR-26a Mediates Adipogenesis of amniotic fluid mesenchymal Stem/Stromal cells via PTEN, Cyclin E1, and CDK6. Stem Cells Dev 26: 482-494, 2017.

53. Qiu W, Fderico L, Naples M, Avramoglu RK, Meshkani R, Zhang J, Tsai J, Hussain M, Dai K, Iqbal J, et al: Phosphatase and tensin homolog (PTEN) regulates hepatic lipogenesis, microsomal triglyceride transfer protein, and the secretion of apolipoprotein B-containing lipoproteins. Hepatology 48: 1799-1809, 2008.

54. Iida S, Ono A, Sayama K, Hamaguchi T, Fujii H, Nakajima H, Namba M, Hanafusa T, Matsuzawa Y and Moriwaki K: Accelerated decline of blood glucose after intravenous glucose injection in a patient with Cowden disease having a heterozygous germline mutation of the PTEN/MMAC1 gene. Anticancer Res 20: 1901-1904, 2000.

55. Zeng T, Zhang CL, Zhao N, Guan MJ, Xiao M, Yang R, Zhao XL, Yu LH, Zhu ZP and Xie KQ: Impairment of Akt activity by CYP2E1 mediated oxidative stress is involved in chronic ethanol-induced fatty liver. Redox Biol 14: 295-304, 2018.

56. Shearn CT and Petersen DR: Understanding the tumor suppressor PTEN in chronic alcoholism and hepatocellular carcinoma. Adv Exp Med Biol 815: 173-184, 2015.

57. Peyrou M, Bourgoin L and Foti M: PTEN in non-alcoholic fatty liver disease/non-alcoholic steatohepatitis and cancer. Dig Dis 28: 236-246, 2010.

58. Slavin J, Jacobs D and Marquart L: Whole-grain consumption and chronic disease: Protective mechanisms. Nutr Cancer 27: 14-21, 1997.

59. Nourhashemi F, Gillette-Guyonnet S, Andrieu S, Ghisolfi A, Ousset PJ, Grandjean H, Grand A, Pous J, Vellas B and Albarede JL: Alzheimer disease: Protective factors. Am J Clin Nutr 71 (Suppl): 643S-649S, 2000.

60. Tokuhira N, Kitagishi Y, Suzuki M, Minami A, Nakanishi A, Ono Y, Kobayashi K, Matsuda S and Ogura Y: PI3K/AKT/PTEN pathway as a target for Crohn's disease therapy (Review). Int J Mol Med 35: 10-16, 2015

61. Barreto R, Kawakita S, Tsuchiya J, Minelli E, Pavasuthipaisit K, Helmy A and Marotta F: Metal-induced oxidative damage in cultured hepatocytes and hepatic lysosomal fraction: Beneficial effect of a Curcumin/Absinthium compound. Chin J Dig Dis 6 : 31-36, 2005.

62. Shehzad A, Qureshi M, Anwar MN and Lee YS: Multifunctional curcumin mediate multitherapeutic effects. J Food Sci 82: 2006-2015, 2017

63. Qiang Z, Meng L, Yi C, Yu L, Chen W and Sha W: Curcumin regulates the miR-21/PTEN/Akt pathway and acts in synergy with PD98059 to induce apoptosis of human gastric cancer MGC-803 cells. J Int Med Res 47: 1288-1297, 2019.

64. Andújar I, Ríos JL, Giner RM and Recio MC: Pharmacological properties of shikonin-a review of literature since 2002. Planta Med 79: 1685-1697, 2013

65. Xie L, Li M, Liu D, Wang X, Wang P, Dai H, Yang W, Liu W, $\mathrm{Hu} \mathrm{X}$ and Zhao M: Secalonic Acid-F, a Novel Mycotoxin, represses the progression of hepatocellular carcinoma via MARCH1 regulation of the $\mathrm{PI} 3 \mathrm{~K} / \mathrm{AKT} / \beta$-catenin signaling pathway. Molecules 24: pii: E393, 2019.

66. Wang H, Chen L, Zhang X, Xu L, Xie B, Shi H, Duan Z, Zhang H and Ren F: Kaempferol protects mice from d-GalN/LPS-induced acute liver failure by regulating the ER stress-Grp78-CHOP signaling pathway. Biomed Pharmacother 111: 468-475, 2019.

67. Xu B, Jiang C, Han H, Liu H, Tang M, Liu L, Ji W, Lu X, Yang X, Zhang Y and Liu Y: Icaritin inhibits the invasion and epithelial-to-mesenchymal transition of glioblastoma cells by targeting EMMPRIN via PTEN/AKt/HIF-1 $\alpha$ signalling. Clin Exp Pharmacol Physiol 42: 1296-1307, 2015.

68. Lee MK, Choi YJ, Sung SH, Shin DI, Kim JW and Kim YC: Antihepatotoxic activity of icariin, a major constituent of Epimedium koreanum. Planta Med 61: 523-526, 1995.

69. Lou K, Yang M, Duan E, Zhao J, Yu C, Zhang R, Zhang L, Zhang M, Xiao Z, Hu W and He Z: Rosmarinic acid stimulates liver regeneration through the mTOR pathway. Phytomedicine 23: $1574-1582,2016$

70. Yoshida H, Okumura N, Kitagishi Y, Nishimura Y and Matsuda S: Ethanol extract of Rosemary repressed PTEN expression in K562 culture cells. Int J Appl Biol Pharm Technol 2: 316-322, 2011.

71. Wang Z, Hu JN, Yan MH, Xing JJ, Liu WC and Li W: Caspase-mediated anti-apoptotic effect of ginsenoside Rg5, a main rare ginsenoside, on acetaminophen-induced hepatotoxicity in mice. J Agric Food Chem 65: 9226-9236, 2017.
72. Sarady JK, Zuckerbraun BS, Bilban M, Wagner O, Usheva A, Liu F, Ifedigbo E, Zamora R, Choi AM and Otterbein LE: Carbon monoxide protection against endotoxic shock involves reciprocal effects on iNOS in the lung and liver. FASEB J 18 854-856, 2004

73. Huo Y, Win S, Than TA, Yin S, Ye M, Hu H and Kaplowitz N: Antcin $\mathrm{H}$ Protects against acute liver injury through disruption of the interaction of c-Jun-N-terminal kinase with mitochondria. Antioxid Redox Signal 26: 207-220, 2017.

74. Qian Y, Guan T, Huang M, Cao L, Li Y, Cheng H, Jin H and Yu D Neuroprotection by the soy isoflavone, genistein, via inhibition of mitochondria-dependent apoptosis pathways and reactive oxygen induced-NF- $\mathrm{B}$ activation in a cerebral ischemia mouse model. Neurochem Int 60: 759-767, 2012.

75. Fan YJ, Rong Y, Li PF, Dong WL, Zhang DY, Zhang L and Cui MJ: Genistein protection against acetaminophen-induced liver injury via its potential impact on the activation of UDP-glucuronosyltransferase and antioxidant enzymes. Food Chem Toxicol 55: 172-181, 2013

76. Stice CP, Xia H and Wang XD: Tomato lycopene prevention of alcoholic fatty liver disease and hepatocellular carcinoma development. Chronic Dis Transl Med 4: 211-224, 2018.

77. Robinett NG, Peterson RL and Culotta VC: Eukaryotic copper-only superoxide dismutases (SODs): A new class of SOD enzymes and SOD-like protein domains. J Biol Chem 293: 4636-4643, 2018

78. Desjardins F, Sekkali B, Verreth W, Pelat M, De Keyzer D, Mertens A, Smith G, Herregods MC, Holvoet P and Balligand JL: Rosuvastatin increases vascular endothelial PPARgamma expression and corrects blood pressure variability in obese dyslipidaemic mice. Eur Heart J 29: 128-137, 2008

79. Chatterjee A, Ronghe A, Padhye SB, Spade DA, Bhat NK and Bhat HK: Antioxidant activities of novel resveratrol analogs in breast cancer. J Biochem Mol Toxicol: 32, 2018 doi: $10.1002 / \mathrm{jbt} .21925$

80. Ribeiro CCD, Silva RM, Campanholo VMLP, Ribeiro DA, Ribeiro Paiott AP and Forones NM: Effects of grape juice in superoxide dismutase and catalase in colorectal cancer carcinogenesis induced by azoxymethane. Asian Pac J Cancer Prev 19: 2839-2844, 2018

81. Rodrigues AD, Scheffel TB, Scola G, Dos Santos MT, Fank B, Dani C, Vanderlinde R, Henriques JA, Coitinho AS and Salvador M: Purple grape juices prevent pentylenetetrazol-induced oxidative damage in the liver and serum of Wistar rats. Nutr Res 33: 120-125, 2013

82. Geeraert B, Crombé F, Hulsmans M, Benhabilès N, Geuns JM and Holvoet P: Stevioside inhibits atherosclerosis by improving insulin signaling and antioxidant defense in obese insulin-resistant mice. Int J Obes (Lond) 34: 569-577, 2010

83. Moselhy SS, Ghoneim MA and Khan JA: In vitro and in vivo evaluation of antimicrobial and antioxidant potential of stevia extract. Afr J Tradit Complement Altern Med 13: 18-21, 2016.

84. Ma N, Abaker JA, Bilal MS, Dai H and Shen X: Sodium butyrate improves antioxidant stability in sub-acute ruminal acidosis in dairy goats. BMC Vet Res 14: 275, 2018.

85. Jin CJ, Engstler AJ, Sellmann C, Ziegenhardt D, Landmann M, Kanuri G, Lounis H, Schröder M, Vetter W and Bergheim I: Sodium butyrate protects mice from the development of the early signs of non-alcoholic fatty liver disease: Role of melatonin and lipid peroxidation. Br J Nutr: 1-12, Nov 23 , (Epub ahead of print)

86. Ren T, Zhu L, Shen Y, Mou Q, Lin T and Feng H: Protection of hepatocyte mitochondrial function by blueberry juice and probiotics via SIRT1 regulation in non-alcoholic fatty liver disease. Food Funct 10: 1540-1551, 2019.

87. Abdulkhaleq FM, Alhussainy TM, Badr MM, Khalil AAA, Gammoh O, Ghanim BY and Qinna NA: Antioxidative stress effects of vitamins $\mathrm{C}, \mathrm{E}$, and $\mathrm{B}_{12}$, and their combination can protect the liver against acetaminophen-induced hepatotoxicity in rats. Drug Des Devel Ther 12: 3525-3533, 2018.

88. Bello RI, Gómez-Díaz C, Burón MI, Alcaín FJ, Navas P and Villalba JM:Enhanced anti-oxidant protection of liver membranes in long-lived rats fed on a coenzyme Q10-supplemented diet. Exp Gerontol 40: 694-706, 2005.

89. Musalmah M, Nizrana MY, Fairuz AH, NoorAini AH, Azian AL, Gapor MT and Wan Ngah WZ: Comparative effects of palm vitamin $\mathrm{E}$ and alpha-tocopherol on healing and wound tissue antioxidant enzyme levels in diabetic rats. Lipids 40: 575-580, 2005. 
90. Aliche-Djoudi F, Podechard N, Collin A, Chevanne M, Provost E, Poul M, Le Hégarat L, Catheline D, Legrand P, Dimanche-Boitrel MT, Lagadic-Gossmann D and Sergent O: A role for lipid rafts in the protection afforded by docosahexaenoic acid against ethanol toxicity in primary rat hepatocytes. Food Chem Toxicol 60: 286-296, 2013.

91. Ide T, Kobayashi H, Ashakumary L, Rouyer IA, Takahashi Y, Aoyama T, Hashimoto T and Mizugaki M: Comparative effects of perilla and fish oils on the activity and gene expression of fatty acid oxidation enzymes in rat liver. Biochim Biophys Acta 1485: 23-35, 2000.

92. Manley S and Ding W: Role of farnesoid X receptor and bile acids in alcoholic liver disease. Acta Pharm Sin B 5: 158-167, 2015.

93. Forsyth CB, Farhadi A, Jakate SM, Tang Y, Shaikh M and Keshavarzian A: Lactobacillus GG treatment ameliorates alcohol-induced intestinal oxidative stress, gut leakiness, and liver injury in a rat model of alcoholic steatohepatitis. Alcohol 43: 163-172, 2009.
94. Bastian WP, Hasan I, Lesmana CRA, Rinaldi I and Gani RA: Gut Microbiota profiles in nonalcoholic fatty liver disease and its possible impact on disease progression evaluated with transient elastography: Lesson learnt from 60 cases. Case Rep Gastroenterol 13: 125-133, 2019.

95. Sharpton SR, Maraj B, Harding-Theobald E, Vittinghoff E and Terrault NA: Gut microbiome-targeted therapies in nonalcoholic fatty liver disease: A systematic review, meta-analysis, and meta-regression. Am J Clin Nutr 110: 139-149, 2019.

This work is licensed under a Creative Commons Attribution-NonCommercial-NoDerivatives 4.0 International (CC BY-NC-ND 4.0) License. 